Research Article

\title{
Structure Optimization of Vibrating Feeder Based on Inertia Release
}

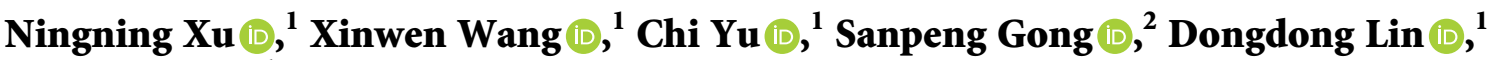 \\ and Xing Su ${ }^{1}$ \\ ${ }^{1}$ School of Chemical and Environmental Engineering, China University of Mining and Technology (Beijing),
Beijing 100083, China
${ }^{2}$ School of Mechanical and Power Engineering, Henan Polytechnic University, Jiaozuo 454003, China \\ Correspondence should be addressed to Xinwen Wang; xinwen.w@cumtb.edu.cn and Sanpeng Gong; 18813089516@163.com
}

Received 2 August 2020; Revised 5 December 2020; Accepted 10 January 2021; Published 30 January 2021

Academic Editor: Chengzhi Shi

Copyright (C) 2021 Ningning Xu et al. This is an open access article distributed under the Creative Commons Attribution License, which permits unrestricted use, distribution, and reproduction in any medium, provided the original work is properly cited.

Reliability is a key factor in the design and manufacture of vibrating feeders. In this paper, a method considering materials force was proposed to optimize the structure of the vibrating feeder. Discrete Element Method (DEM) was used to couple the materials force and the excitation force based on the phase characteristics of the vibrating feeder, and Finite Element Method (FEM) was used to analyze the vibrating feeder structure based on the inertia release method. In order to reduce the stress on the beam of the vibrating feeder, three structural improvement schemes were proposed, which were constructing a statically indeterminate structure, increasing the width of the rib-stiffened plates on the beam, and increasing the internal spacing of the beam. Then, these three schemes were compared using the FEM. Finally, the response surface method was used to optimize the width of the inner and outer rib-stiffened plates. The research results showed that when the vibrating feeder moved close to the highest point, the materials force reached the peak. The maximum first principal stress occurred at the middle and both ends of the vibrating feeder beam under the joint action of the excitation force and the materials force. The first principal stress value of the beam was most significantly decreased by increasing the width of the rib-stiffened plates on the beam of three optimization schemes. The optimal increment of rib-stiffened plate width was $30 \mathrm{~mm}$ with the maximum first principal stress value reduced by $40.12 \%$.

\section{Introduction}

Vibrating feeder is a kind of feeding equipment widely used in metallurgy, coal, electric power, and chemical industries. It is used with other types of equipment together to realize the automatic operation of feeding, batching, quantitative packaging, and other processes [1]. Due to the fact that the vibrating feeder is used to convey materials, its structure is subject to a large dynamic load, which can easily cause local elastic deformation and lead to fatigue failure in the structure. With the development of large-sized designed vibrating feeders, the alternating load from the exciting force, the inertial load of steel structure, and the impact load from materials have increased significantly, which has aggravated the structural damage of vibrating feeders. Beams of vibrating feeders connecting side panels and bed surface of vibrating feeders are the main components that suffer from various loads; therefore, damage and even fractures often occur in this place firstly, which results in the feeder not working properly, severely restricting production and causing huge economic losses. Therefore, it is necessary to optimize the structure of the vibrating feeder, improving its reliability to ensure the smooth progress of the operation process in industrial production.

Weingerl and Schaflinger considered the energy and momentum transfer on the vibrating feed bed surface, established the theory of particle flow equation on the vibrating feed bed surface, and verified it with a high-speed camera [2]. Han and Lee studied the material jumping motion in the vertical vibration conveyor and reported that when the external conditions changed, the material would have bifurcation motion or even chaotic motion [3]. 
Ashrafizadeh and Ziaei-Rad considered the influence of material shape on its movement and used rectangular blocks instead of mass points to study material movement using the DEM [4]. Kong et al. established a sliding and jumping motion model of materials based on Coulomb's law of friction and collision principle, and the model is used to analyze the changes of material motion under different vibration conditions. It is found that there is forward sliding or even reverse sliding in the process of material jumping movement, and the sliding effect has a certain influence on the movement of material [5]. Afanas'ev1 and Suslov studied the vibration transport rate of the free-flowing rock mass. The energy consumption and reasonable system parameters, especially the oscillation frequency, amplitude, and asymmetry coefficient, are determined [6]. Xin et al. established a mechanical model of inclined vibrating feeder with the material binding coefficient based on considering the nonlinear force of materials and obtained the correlation between material binding coefficient and inclination, amplitude, and frequency of working face based on the solution and analysis results of the model [7]. Zhang designed an equal thickness vibrating feeder for achieving radial equal thickness distribution of materials. Through field test analysis of dynamic response parameters of the feeder, the vibration characteristics such as amplitude, frequency, vibration track, and vibration direction angle of base and sieve of the vibrating feeder were obtained [8]. Most of these studies focused on the dynamic characteristics of material movement or vibration angle and amplitude of the vibrating feeder; however, there were few types of research on the structure optimization and reliability of the vibrating feeder.

Similar to vibrating feeders, vibrating screens are subject to a large dynamic load during the vibration process. To improve the reliability of large vibrating screens, Zhao et al. presented a new design of a hyperstatic net-beam structure and analyzed dynamic characteristics of it, based on the FEM, and the new screen proves to have much higher structural strength with an enhanced dynamic behavior [9]. Su et al. proposed an improved scheme of beam sections for large-scale vibrating screen structure based on static analysis and dynamic analysis, where ameliorated stress distribution of the vibrating screen is in the working process, and the fatigue life is increased [10]. Baragetti presented a structural solution for high loaded vibrating screens with new modified side plates and studied the behavior of the original and modified structure using theoretical and numerical models [11]. Peng et al. conducted a systematic mechanics analysis of the beam structures and improved the design method considering bending and random vibration [12]. Yang decoupled the complex space motion of the vibrating screen and gave the dynamic decoupling condition of the vibrating screen. He used the BP neural network to model the relationship between design variables and elastic deformation of the vibrating screen [13]. The vibrating equipment receives the reaction force of the materials in the process of screening and conveying the materials, that is, the materials force. However, the above optimization of the structure of the vibrating screen did not consider the effect of the materials force. In this paper, the EDEM was used to couple the materials force and the excitation force on the basis of the phase characteristics so that the impact of the materials force was fully considered in the optimization process.

The inertia release method $[14,15]$ was often used to calculate the stress distribution of structures such as aircraft, automobiles, and ships. The vibrating feeder is in a noncompletely constrained state during the vibration process. In this paper, the inertia release method was used to analyze the stress of the vibrating feeder during the operation process. This made it possible to use statics to analyze the transient stress of the vibrating feeder when it was running reducing the complexity of the dynamic analysis of the vibrating feeder [16]. This also avoided the influence of the boundary conditions of fixed constraints on the stress calculation results in the static analysis, making the constraints of the finite element analysis consistent with the actual working conditions.

In summary, this paper provided a structural optimization method for vibrating feeder, which was suitable for considering materials force on the vibrating feeder.

\section{Principle of Inertia Release}

Inertia release is a method that constructs a balanced force system through inertial force by calculating the acceleration of the structure under the action of an unbalanced external force. It can simulate the static response of unconstrained or underconstrained systems. Due to the limitation of the design load calculation method and the cumulative error of numerical calculation, it is extremely difficult to obtain an absolute self-balanced force system for the complicated structure of vibrating feeder, but a self-balancing force system can be constructed by the static and dynamic method. The FEM was used to establish the undamped static and dynamic balance equation as shown in equation (1):

$$
\{F\}+[M]\{\ddot{\delta}\}=0
$$

where $\{F\}$ presents the nodal load vector composed of all nodal load components and $\{\ddot{\delta}\}$ is the node acceleration vector composed of all node acceleration components. $[M]=$ $\int_{\Omega} \rho N^{T} N \mathrm{~d} \Omega$ stands for the mass matrix, where $N$ is the shape matrix, $\rho$ is the density, and $\mathrm{d} \Omega$ is the volume of the unit.

The node acceleration required at each node to maintain balance can be obtained by solving and calculating the above equation. Then the inertial force of each node can be calculated. A self-balancing force system can be constructed by adding the inertial force as an external force to the node of the finite elements $[17,18]$.

\section{Coupling of Materials Force and Excitation Force}

The vibrating feeder is affected by its excitation force as well as the materials force on its bed surface. Generally, the materials force is so large when there is a stock bin above the feeder or the material layer is very thick that the impact of materials force cannot be ignored. Therefore, the influence of the materials force should be considered in the stress 
analysis of the vibrating feeder according to actual operating conditions. It is difficult to obtain the stress of the vibrating feeder under the simultaneous action of excitation force and materials force using the DEM or the FEM only. Therefore, an effective method is to couple the DEM and FEM for analysis. In this paper, the materials force on the vibrating feeder was calculated using the EDEM software. The phase relationship between the materials force and the motion of the vibrating feeder can be obtained by EDEM, and the phase relationship between the exciting force and the motion of the vibrating feeder can be obtained by equation (4). Therefore, the phase relationship between the materials force and excitation force can be obtained indirectly, and then the excitation force and materials force can be set in ANSYS as an external load to realize the coupling of excitation force and materials force.

3.1. Setting and Calculation of EDEM. The vibrating feeder is usually used in the feed system. The feeding process was simulated by EDEM software to calculate the phase relationship between the materials force and the motion of the vibrating feeder, as presented in Figure 1. The feed system model in the simulation included a stock bin, a sluice gate, and a vibrating feeder. The coal was selected as conveyed materials in this work. Material parameters and interaction parameters set in EDEM are shown in Table 1.

Affected by the pressure of stock bin bottom and the variation of thickness of the material layer, the materials force in each area of the bed surface also changes along the conveying direction while the materials force on the bed surface perpendicular to the material conveying direction is relatively uniform, while only the materials force difference between the heavy-load area and the material-layer area was large. Therefore, the bed surface was divided into two areas according to the heavy-load area and the material-layer area to simulate and calculate the materials force in each area on the bed surface of the vibrating feeder that was set as the external load to calculate the structural stress of the feeder. The heavy-load area was further subdivided into two areas and the material-layer area was further subdivided into four areas, and then the above simulation calculation process was performed again. It was found that the calculation results of dividing into six areas were basically the same as dividing into two areas of material-layer load and heavy-load load comparing the finite element stress calculation results, indicating that the division of two materials force areas can accurately analyze the influence of materials force on the stress of the feeder structure. However, in this work, in order to obtain more detailed material distribution results, the vibrating feeder bed surface was divided into six areas along the conveying direction where areas 1 to 4 were the load areas of the material layer, and areas 5 and 6 were the heavyload areas, as shown in Figure 1.

The time-domain curve of the materials force in area 6 is shown in Figure 2. It showed the materials force variation in the whole process of loading material into the vibration feeding system until the stock bin bottom pressure was stable and then the vibrating feeder started to vibrate. Due to the material arching effect [19], the stock bin bottom pressure reached a dynamically stable value in actual production. Therefore, to obtain the same stable stock bin bottom pressure during the simulation calculation, the particle factory was set to produce enough particles to make the stock bin bottom pressure reach a steady-state in the simulation. After the stock bin bottom pressure stopped increasing, the vibrating feeder was set to vibrate at a frequency of $16 \mathrm{~Hz}$, a double amplitude of $3.4 \mathrm{~mm}$, an inclination of $10^{\circ}$, and a vibration direction angle of $30^{\circ}$. It can be seen from Figure 2 that the materials force fluctuated violently when the vibrating feeder started to vibrate and tended to be dynamically stable after $12 \mathrm{~s}$.

3.2. Coupling Method. At $13.00 \mathrm{~s}$, the vibration response of the materials force reached the dynamic stability state (an approximate periodically changing state). The materials force in each area is shown in Figure 3. The upper curve is the displacement curve of the vibrating feeder, and the upward direction of the vibrating feeder moving is specified as the positive direction. The time-domain correspondence between the movement displacement of the vibrating feeder body and the materials force in each area of the material can be obtained from Figure 3. Since the exciting force of the feeder and the force of the materials changed periodically, there was a phase relationship between the two forces. Therefore, before setting the external load of the feeder in ANSYS software, it was necessary to know the phase relationship between the exciting force and the material force to couple the two forces together. The EDEM software was used to simulate the feeder conveying materials, and the resulting output by the EDEM software only included the phase relationship between the material force and the motion of the vibrating feeder. The phase relationship between the feeder motion and the exciting force can be obtained through equation (2), so that we can indirectly obtain the phase relationship between the exciting force and the materials force through the feeder motion phase.

The vibration of the vibrating feeder is forced vibration excited by a harmonic inertia force. The phase difference between the simple harmonic excitation force and the displacement response of the vibrating feeder is $\varphi$, which is calculated as equation (2) [20]:

$$
\varphi=\arctan \frac{2 \zeta r}{1-r^{2}},
$$

where $\zeta$ represents the damping ratio and $r$ stands for the frequency ratio.

In this work, the working frequency point of the vibrating feeder was located in the far resonance zone $\zeta \approx 0.05$, $r \approx 4$. According to equation $(2), \varphi \approx 180^{\circ}$. It meat that the vibrating feeder displacement response phase was about $180^{\circ}$ behind the excitation force. Based on this phase characteristic, combined with the time-domain correspondence between the materials force and the feeder displacement obtained by the EDEM simulation shown in Figure 3, the phase relationship between the materials force and the exciting force can be obtained. Take the process of obtaining 




FIgURE 1: EDEM simulation of vibrating feeding system.

TABle 1: Parameters set in EDEM.

\begin{tabular}{lcc}
\hline Parameter type & Material & Model \\
\hline Density $\left(\mathrm{kg} \mathrm{m}^{-3}\right)$ & 1,600 & 7,850 \\
Shear modulus $(\mathrm{GPa})$ & 0.03 & 10 \\
Poisson's ratio & 0.2 & 0.3 \\
Coefficient of restitution & 0.03 & 0.08 \\
Coefficient of static friction & 1 & 0.5 \\
Coefficient of rolling friction & 0.1 & 0.01 \\
\hline
\end{tabular}

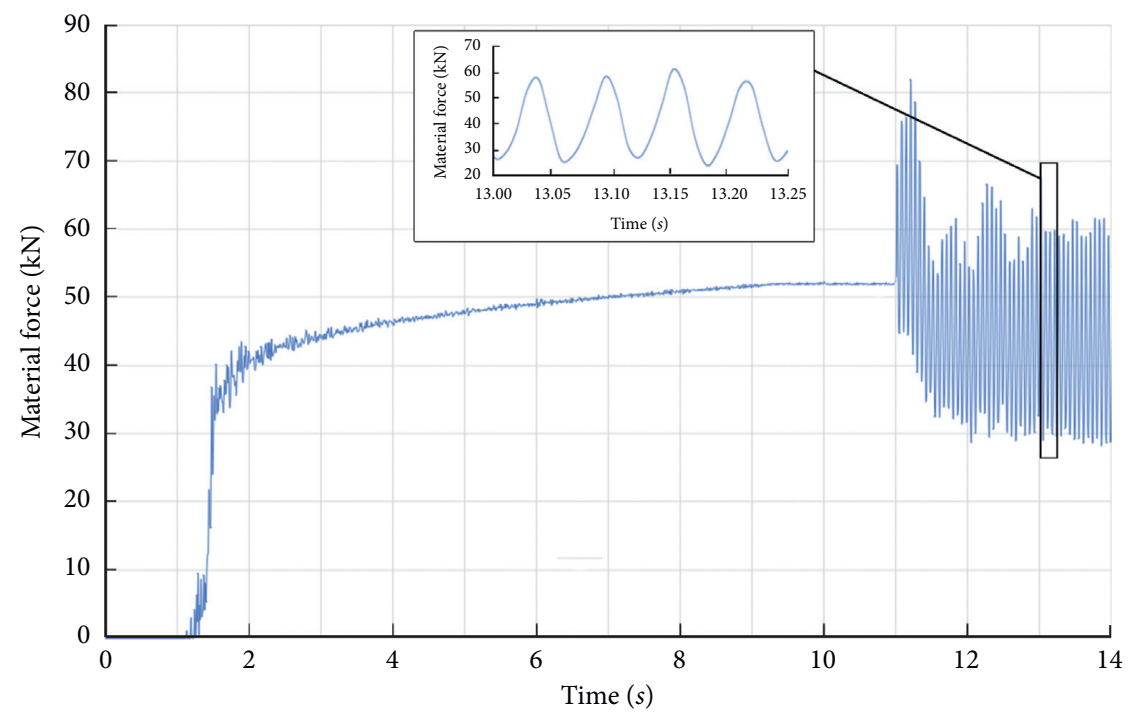

Figure 2: Time-domain curve of materials force on vibrating feeder surface.

the phase between the excitation force of the same direction and forward and the materials force of the vibrating feeder as an example to further illustrate the method of obtaining the phase relationship between the excitation force and the materials force. The red dotted line corresponds to the vibrating feeder displacement to the highest point at the moment as shown in Figure 3. According to the conclusion that the displacement response phase of the feeder lags behind the excitation force phase by $180^{\circ}$, it can be known that the direction of the exciting forces is the same direction and backward at this moment. It is obvious from Figure 3 that the materials force reached the maximum value in the 


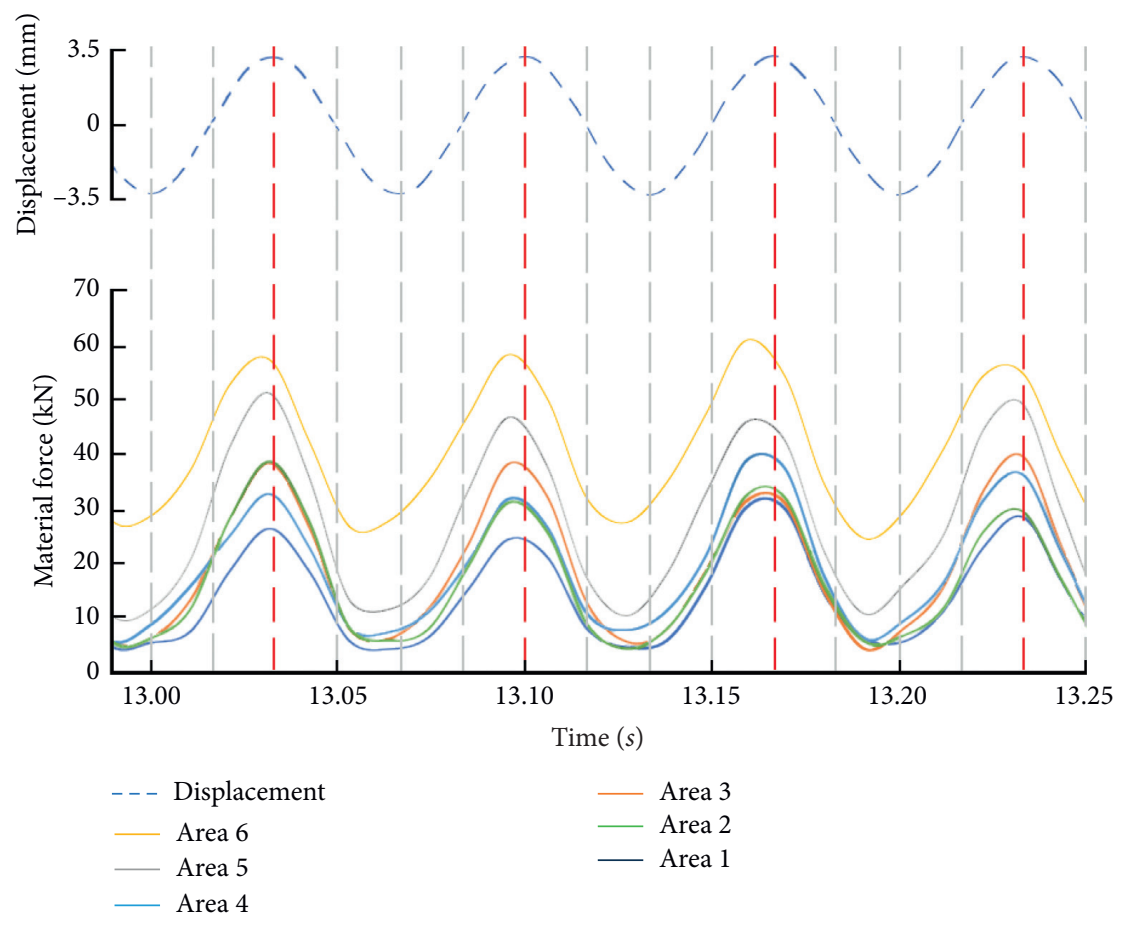

FIGURE 3: The time-domain corresponding relationship between vibrating feeder motion and materials force.

cycle when approaching this moment. Namely, the materials force reached the largest when the upward movement of the vibrating feeder was about to reach the highest point.

Using this method, the phase relationship between the exciting force and the materials force at other moments could be obtained. Further analysis of the curve in Figure 3 could lead to the following conclusions that when the vibrating feeder displacement was close to the lowest point, the materials force reached the minimum value. Due to the smaller thickness of the materials layer, the materials force was less in areas 1-4, while areas 5 and 6 were located directly below the stock bin, so they carried most of the stock bin bottom pressure.

\section{Model Meshing and Evaluation of Meshing}

As shown in Figure 4, the shape of the vibration motor that did not participate in structural optimization was simplified in the simplified model of the body of the vibrating feeder. According to the parameters such as the distance between the axis of the original vibration motor and the side plate and the distance between the mounting seat, the point of action of the excitation force, and vibrating motor mass, the vibration motor model was simplified so that the excitation force generated by it remained unchanged. The finite element model of the vibrating feeder was imported in ANSYS, as shown in Figure 4 . The main structure adopted $10 \mathrm{~mm}$ meshes, and the local refinement mesh size was $3 \mathrm{~mm}$. The tetrahedral mesh type was selected. The finite element model included 856185 units and 1565867 nodes.

Meshing is the key link to finite element analysis, and the quality of the meshes directly affects the correctness of the calculation results. The closer the element is to the regular polyhedron, the more accurate the finite element analysis result obtained from its calculation. In this paper, the orthogonal quality was used to evaluate the quality of the element. Each element was analyzed according to equations (3) and (4). The minimum $Y_{i}$ value obtained is the orthogonal quality coefficient.

$$
\begin{aligned}
& Y_{1}=\frac{\vec{A}_{i} \cdot \vec{f}_{i}}{\left|\vec{A}_{i}\right| \cdot\left|\vec{f}_{i}\right|}, \\
& Y_{2}=\frac{\vec{A}_{i} \cdot \vec{c}_{i}}{\left|\vec{A}_{i}\right| \cdot\left|\vec{c}_{i}\right|},
\end{aligned}
$$

where $\vec{f}_{i}$ is the vector from the centroid to each of its faces, $\vec{c}_{i}$ is the vector from the centroid to the centroid of each adjacent element, and $\vec{A}_{i}$ is the normal vector of its face.

The calculated orthogonal quality coefficient is between 0 and 1: the closer to 1 , the better, and the closer to 0 , the worse. Generally, orthogonal quality coefficients between 0.25 and 0.75 are considered acceptable meshes, below 0.25 are poor quality meshes, and above 0.75 are good quality meshes.

The orthogonal quality histogram in this finite element analysis is shown in Figure 5. It can be seen from the figure that the mesh element quality coefficient is basically above 0.75 . The distribution of elements with a mesh quality coefficient below 0.25 was selected and displayed in the model as shown in Figure 6. It can be seen from the figure that there are few low-quality meshes on the beam, which is about $1 / 3$ of the total number of low-quality meshes. The low-quality meshes were not focused on the area of interest, so the lowquality meshes had little effect on the finite element analysis 


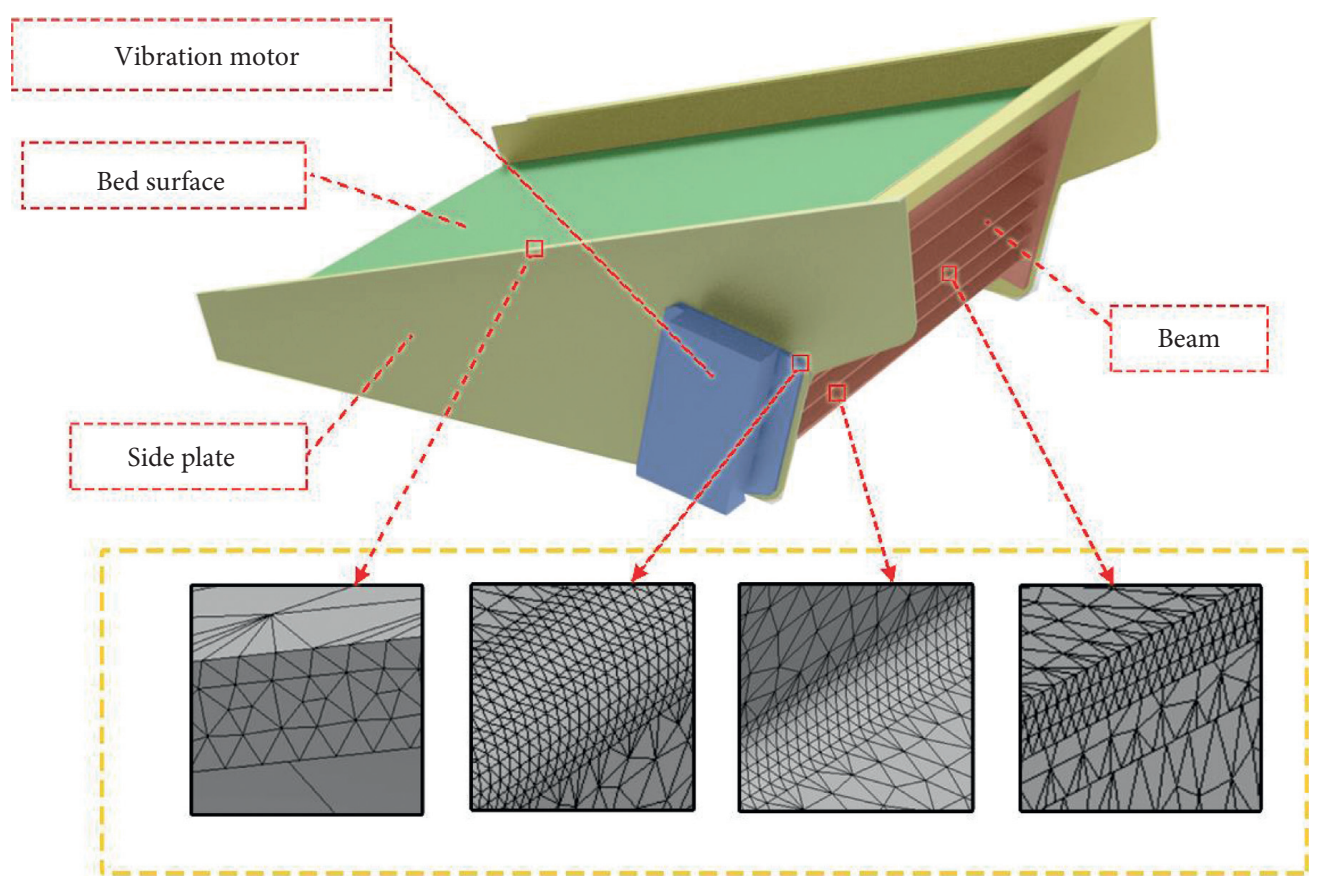

Local refinement meshes

Figure 4: Local refinement meshes and the simplified model of vibrating feeder.



FIGURE 5: Orthogonal quality of meshes bar chart.

results of the beam. The mesh quality met the requirements of the finite element analysis.

\section{Stress Analysis and Optimization}

5.1. Stress Analysis. The vibrating feeder performs reciprocating vibration under periodic excitation force, and the inertial release method was used to enable the static force analysis of the vibrating feeder at a certain moment during the vibration process. Therefore, it was necessary to select a certain moment in the vibration process of the vibrating feeder as the analysis object. In this paper, four moments within one vibration period of the vibrating feeder were selected for analysis. As shown in Figure 7, four moments corresponding to different directions of the exciting force of the vibrating feeder were selected. The green arrow represents the direction of material movement, and the red arrow represents the direction of the exciting force. These four moments correspond to the maximum amplitude and maximum speed of the vibrating feeder respectively. When the direction of the exciting force was the same and forward, the materials force almost reached the maximum. The reverse inward and reverse outward directions of the exciting force corresponded to the situation where the pressure and the pulling force formed by the exciting force were maximum. Therefore, these four moments could represent the limit conditions of the vibrating feeder during the reciprocating vibration process. According to the coupling method of the materials force and the exciting force in Section 3, the value of the materials force in each area of the vibrating feeder bed surface at these four moments was obtained, as shown in Table 2. 


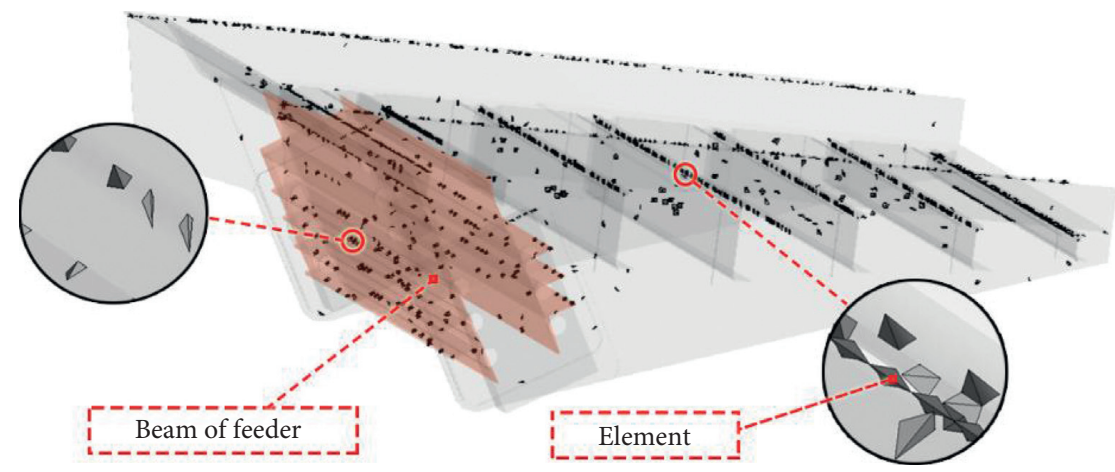

Figure 6: Meshes spatial distributions with the orthogonal quality coefficient of less than 0.25 .
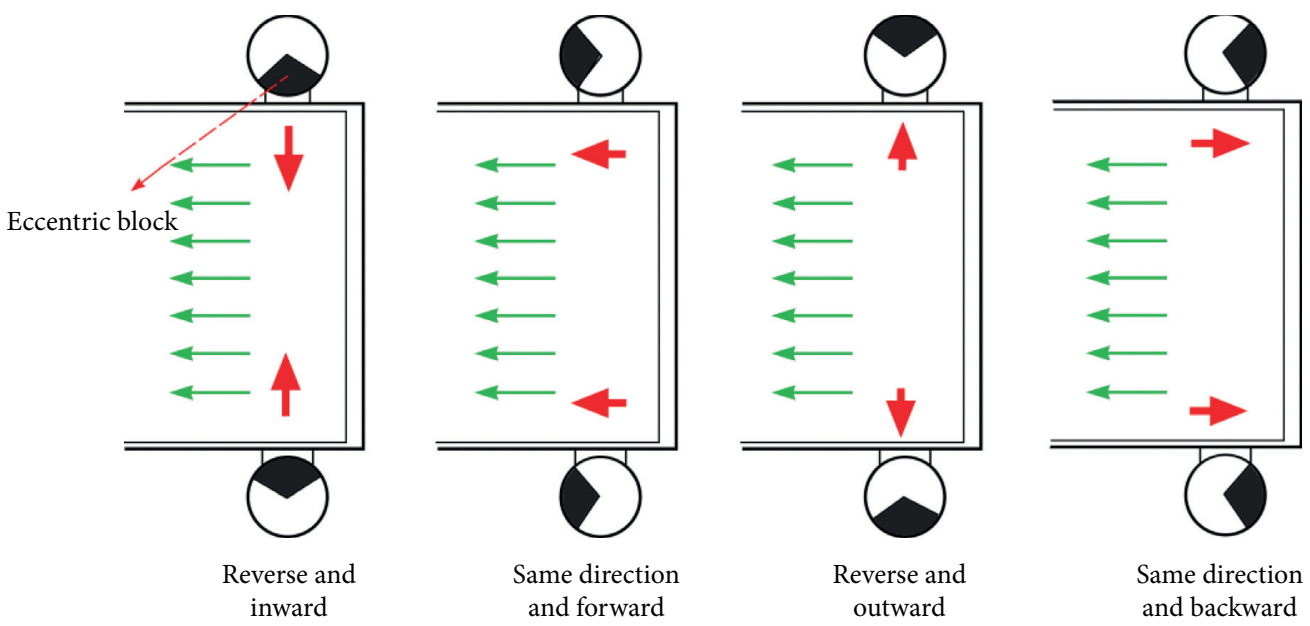

Same direction and backward

FIgURE 7: Exciting force directions at four special moments.

TABLE 2: Material forces in different areas corresponding to the four exciting force directions.

\begin{tabular}{|c|c|c|c|c|c|c|}
\hline $\begin{array}{l}\text { Exciting force } \\
\text { direction }\end{array}$ & $\begin{array}{c}\text { Materials force in } \\
\text { area } 1(N)\end{array}$ & $\begin{array}{c}\text { Materials force in } \\
\text { area } 2(N)\end{array}$ & $\begin{array}{c}\text { Materials force in } \\
\text { area } 3(N)\end{array}$ & $\begin{array}{c}\text { Materials force in } \\
\text { area } 4(N)\end{array}$ & $\begin{array}{c}\text { Materials force in } \\
\text { area } 5(N)\end{array}$ & $\begin{array}{c}\text { Materials force in } \\
\text { area } 6(N)\end{array}$ \\
\hline $\begin{array}{l}\text { Same direction } \\
\text { and forward }\end{array}$ & 30,018 & 13,179 & 9,087 & 8,351 & 7,020 & 5,101 \\
\hline $\begin{array}{l}\text { Same direction } \\
\text { and backward }\end{array}$ & 55,308 & 47,800 & 36,912 & 34,943 & 27,863 & 26,900 \\
\hline $\begin{array}{l}\text { Reverse and } \\
\text { outward }\end{array}$ & 45,011 & 31,098 & 20,130 & 19,201 & 18,967 & 12,099 \\
\hline $\begin{array}{l}\text { Reverse and } \\
\text { inward }\end{array}$ & 31,215 & 19,031 & 13,144 & 11,806 & 11,310 & 9,712 \\
\hline
\end{tabular}

The vibrating feeder loads were set according to all the above parameters. The load setting in Workbench when the excitation force is forward is shown in Figure 8, including gravity, excitation force, and materials force. Structural steel was selected as the model material. At these four moments shown in Figure 7, the boundary conditions need to be set separately and then calculated separately. The inertial release environment was set in Workbench. The first principal stress cloud diagram of the calculation results is shown in Figures $9(\mathrm{a})-9(\mathrm{~d})$. The arrows in the figure indicate the direction of the excitation force.
It can be seen from the first principal stress cloud diagrams at four moments in Figure 9 that no obvious stress concentration was formed on the vibrating feeder bed surface since the materials force was evenly distributed on the bed surface. As shown in Figure 7, the values of the first principal stress on the vibrating feeder were different due to the different direction of the exciting force and the value of the materials force at four moments. However, under the combined action of the materials force and the excitation force, the maximum value of first principal stress was all located at the beam of the vibrating feeder at these four 



FiguRE 8: Boundary condition setting.
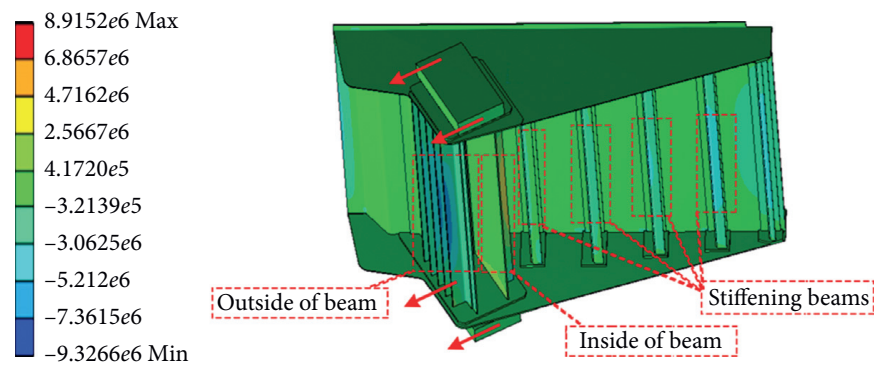

(a)
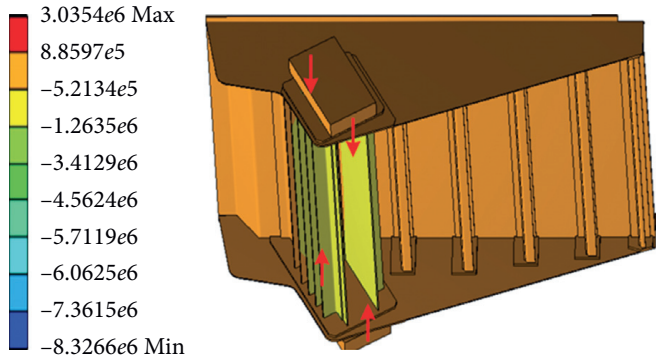

(c)
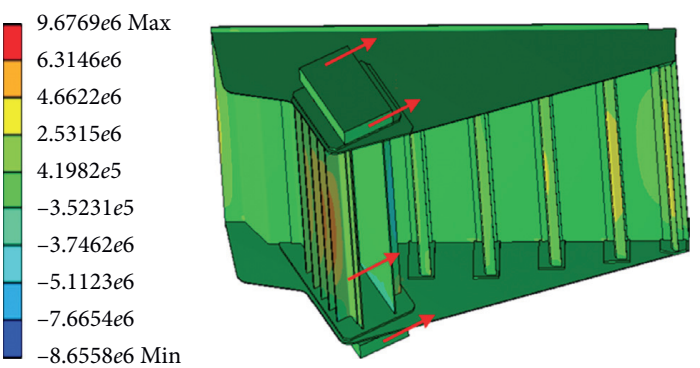

(b)
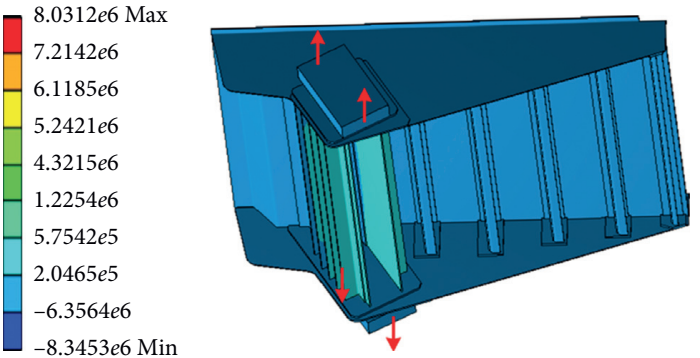

(d)

Figure 9: First principal stress cloud of vibrating feeder at four special moments in one cycle: (a) same direction and backward; (b) same direction and forward; (c) reverse and inward; (d) reverse and outward.

moments. The first principal stress at other locations such as the stiffening beam was large but far less than the first principal stress at the beam. Therefore, it was necessary to optimize the design of the beam to improve the life and reliability of the vibrating feeder beam.

It can be seen from Figure 9 that when the exciting force was in the same direction and forward, the maximum value of the first principal stress of the vibrating feeder beam was $9.6766 e+6 \mathrm{~Pa}$ which was the maximum of the four moments. This was because there was no other tensile structure except for the rib-stiffened plates at the outside of the beam when the excitation force was forward. The first principal stress on the beam rib-stiffened plates was the maximum stress. Therefore, this work aimed to reduce the first principal stress at the moment when the exciting force was in the same direction and forward.

The cross section of the stress cloud diagram of the beam when the excitation force is in the same direction and 
forward was shown in Figure 10. Analysis of the first principal stress calculation result cloud shows that the point of maximum stress appears at the middle and both ends of the vibrating feeder beam. Positive values represent tensile stress and negative values represent compressive stress. As can be seen from Figure 10, when the excitation force was forward, the middle part of the outer rib-stiffened plates of the feeder beam was stretched, the two ends of the ribstiffened plates were extruded, the middle part of the inside was extruded, and the two ends were stretched. The reason for this result was that the bending moment formed by the exciting force causes the feeder beam to bend and side plate extruded rib-stiffened plates at both ends of the beam.

5.2. Structural Optimization. Aiming at the problems of uneven loading of the vibrating feeder beam structure and obvious stress concentration, the following solutions were given, as shown in Figure 11.

Scheme 1: Two stiffening beams were added to the rear end of the vibrating feeder to form the statically indeterminate structure [21]. The tensile structure was increased on the outside of the beam to reduce the stress of the beam.

Scheme 2: According to the mechanical properties of materials, where the point on the beam section is farther away from the neutral axis when the beam is bending, the greater the normal stress is [22], a structural improvement scheme was proposed to widen the rib-stiffened plates located far away from the neutral axis of the beam by $20 \mathrm{~mm}$.

Scheme 3: Similarly, according to the material mechanic's characteristics of the beam, a structural improvement scheme increasing the distance between two plates of the beam by $40 \mathrm{~mm}$ was proposed so that plates were far from the neutral axis of the beam.

The structure of the vibrating feeder was improved according to the above schemes. Then, the finite element analysis was carried out on the improved structure. As shown in Figure 12, the stress cloud diagrams of the beam after the optimization according to the above optimization schemes were obtained. The comparison among stress cloud diagrams of three kinds of structures after the improvements shown in Figure 12 has revealed the following conclusions. The first principal stress of the beam of Scheme 1 drops more obviously except the first principal stress at the two ends of the outer rib-stiffened plates near the stiffening beams increases. This was because additional stiffening beams pulled tight the two side plates to prevent the deformation of the beam, thereby increasing the local stress. The first principal stresses of the beams after structural optimization according to Schemes 2 and 3 have both decreased overall, while the maximum value of the first principal stress value of Scheme 2 was less than that of Scheme 3. Therefore, Scheme 2 was the best optimization scheme.

The reduction rates of the maximum value of the first principal stress of Schemes 1-3 were calculated according to equation (5). The reduction rates of the maximum value of first principal stress of Schemes $1-3$ are $-44.13 \%$ (indicating that the maximum value of first principal stress value does not decrease but increases), 25.63\%, and 25.43\%, respectively.

$$
\gamma=\frac{\alpha_{0}-\alpha_{n}}{\alpha_{0}} \times 100 \%,
$$

where $\alpha_{0}$ stands for the maximum value of the first principal stress of the original structure, $\alpha_{n}$ is the maximum value of the first principal stress of structures of scheme $n, n=1,2,3$, and $\gamma$ represents the stress reduction rate.

5.3. Response Surface Optimization Design. The response surface is a method that finds the relationship between design variables and response variables through regression analysis. It uses experimental design theory to determine the set of design points and then conducts experiments to obtain response surface models of objective functions and constraint functions to predict nonobjective functions. This is an approximate method based on statistical experimental design [23]. In this paper, simulation test points were designed using the central composite design (CCD) method. Then, the best design parameters could be sought based on the response value within the set boundary conditions. The simulation calculation workload of the optimization process could be effectively reduced using CCD.

To obtain the optimal value of the rib-stiffened plate increase, the increase in the length of the rib-stiffened plate on both sides was taken as the design variable, and the maximum value of first principal stress and maximum strain value were taken as the objectives of multiobjective optimization. The mathematical model was as follows:

$$
\begin{aligned}
& \min \delta(X), \min \sigma(X), \\
& \delta(X) \leq \delta_{0}, \sigma(X) \leq \sigma_{0}, \\
& X=\left(x_{1}, x_{2}, \ldots, x_{i}\right)^{T}, \quad X \in \Omega, \\
& 0 \mathrm{~mm}<x_{i}<30 \mathrm{~mm},
\end{aligned}
$$

where $X$ is the design variable, $\delta(X)$ stands for the maximum deformation after optimization, $\delta_{0}$ represents the maximum deformation before optimization, $\sigma(X)$ is the maximum stress after optimization, $\sigma_{0}$ represents the maximum stress before optimization, $\Omega$ stands for the design scope, the lower limit of the design variable is limited to $0 \mathrm{~mm}$, the upper limit of the design scope is limited to $30 \mathrm{~mm}$ by the structural space, and $n$ is the number of design variables, $n=2$, which is the increasing amount in the width of the inner rib-stiffened plates and the outer rib-stiffened plates.

Finite element calculation was performed based on the 17 test points obtained using CCD as shown in Table 3 . Regression calculations were performed based on the data obtained in Table 3 to obtain regression models regarding maximum stress and maximum deformation. The comparison between the observed value of the experimental point and the predicted value of the regression model is shown in Figure 13. The data point composed of the 




Figure 10: First principal stress cloud diagram of vibrating feeder with exciting force forward.

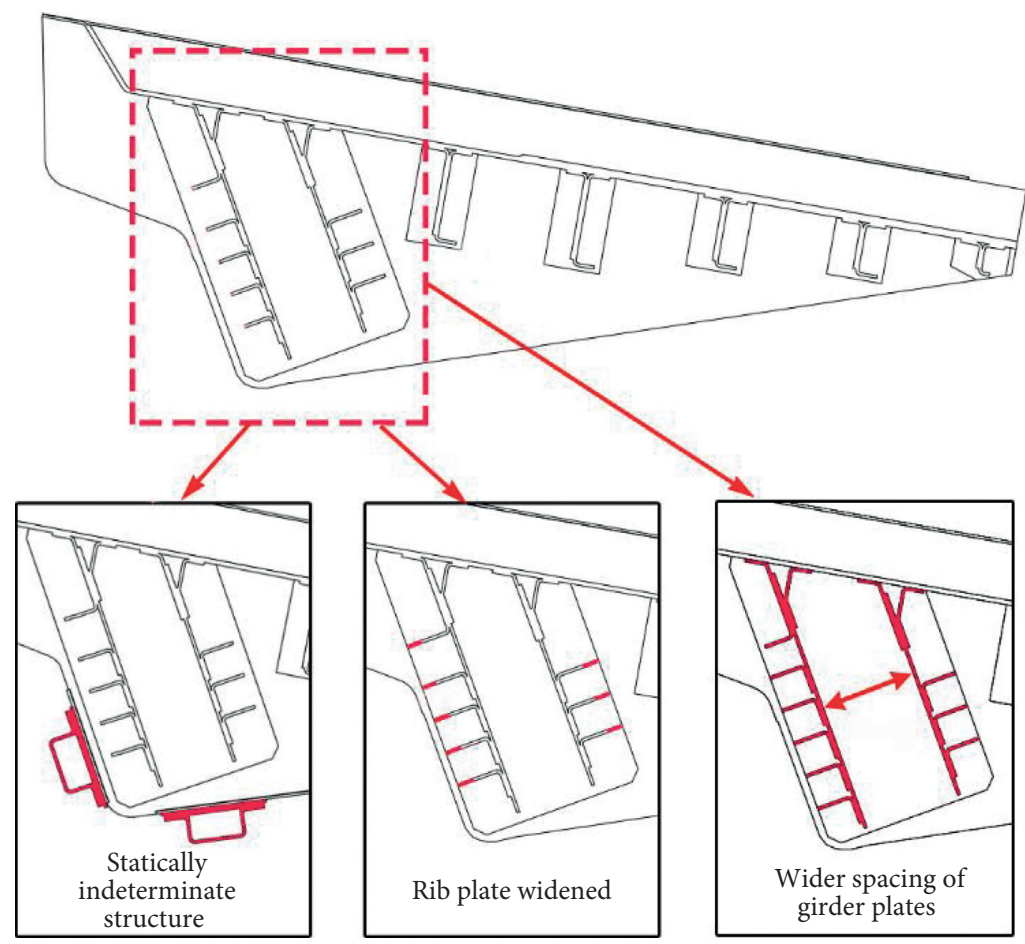

Figure 11: Structural optimization scheme.

observed value of the experimental point and the predicted value of the regression model is basically located on a straight line with a slope of 1 . The determination coefficient $R^{2}$ of the two models and the correction decision coefficient are 0.9427 and 0.9952 , respectively, which are both close to 1 , indicating that the fitting model of maximum stress and maximum deformation has high reliability. According to the analysis results of the regression model, a $3 \mathrm{D}$ response surface graph of various factors that interact with each other was drawn, as shown in Figure 14.

It can be seen from the above two three-dimensional curved surfaces that the maximum value of the first principal stress and the maximum deformation decreased with the increase in the width of the rib-stiffened plates on both sides. The peak value of the maximum deformation caused by the increase in the inner rib-stiffened plate width by $20 \mathrm{~mm}$ and 


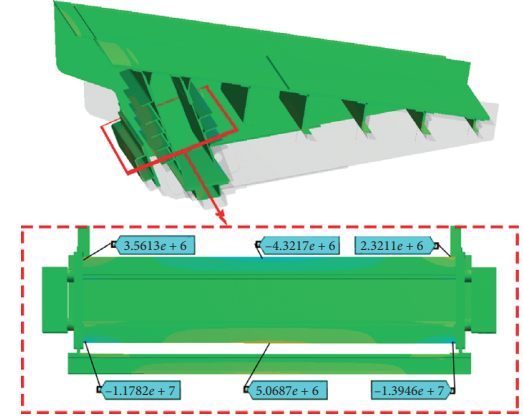

(a)

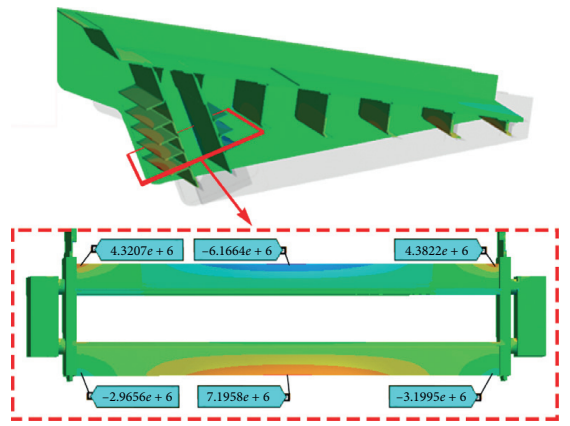

(b)

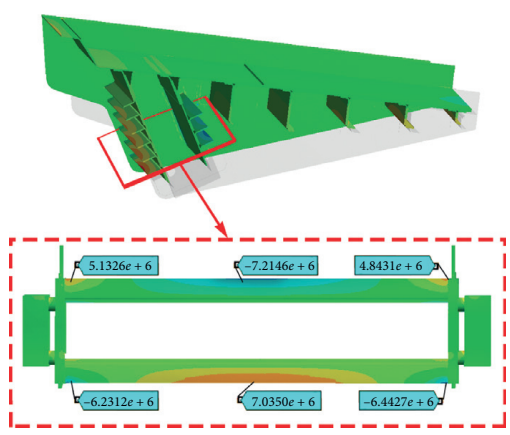

(c)

Figure 12: Stress cloud diagram of the beam of vibrating feeder after optimization. (a) Scheme 1; (b) Scheme 2; (c) Scheme 3.

Table 3: Central composite design test point.

\begin{tabular}{lcccc}
\hline Serial number & Outside rib increment $(\mathrm{m})$ & Inside rib increment $(\mathrm{m})$ & Maximum stress value $(\mathrm{MPa})$ & Maximum deformation $(\mathrm{mm})$ \\
\hline 1 & 0.01550 & 0.01550 & 7.06 & 0.773 \\
2 & 0.00100 & 0.01550 & 7.54 & 0.791 \\
3 & 0.00825 & 0.01550 & 7.37 & 0.784 \\
4 & 0.03000 & 0.01550 & 6.89 & 0.742 \\
5 & 0.02275 & 0.01550 & 7.01 & 0.776 \\
6 & 0.01550 & 0.00100 & 7.66 & 0.813 \\
7 & 0.01550 & 0.00825 & 7.21 & 0.824 \\
8 & 0.01550 & 0.03000 & 6.46 & 0.744 \\
9 & 0.01550 & 0.02275 & 6.57 & 0.757 \\
10 & 0.00100 & 0.00100 & 9.67 & 0.846 \\
11 & 0.00825 & 0.00825 & 8.31 & 0.803 \\
12 & 0.03000 & 0.00100 & 8.49 & 0.815 \\
13 & 0.02275 & 0.00825 & 7.71 & 0.856 \\
14 & 0.00100 & 0.03000 & 7.26 & 0.740 \\
15 & 0.00825 & 0.02275 & 7.59 & 0.814 \\
16 & 0.03000 & 0.03000 & 5.79 & 0.784 \\
17 & 0.02275 & 0.02275 & 6.37 & 0.776 \\
\hline
\end{tabular}

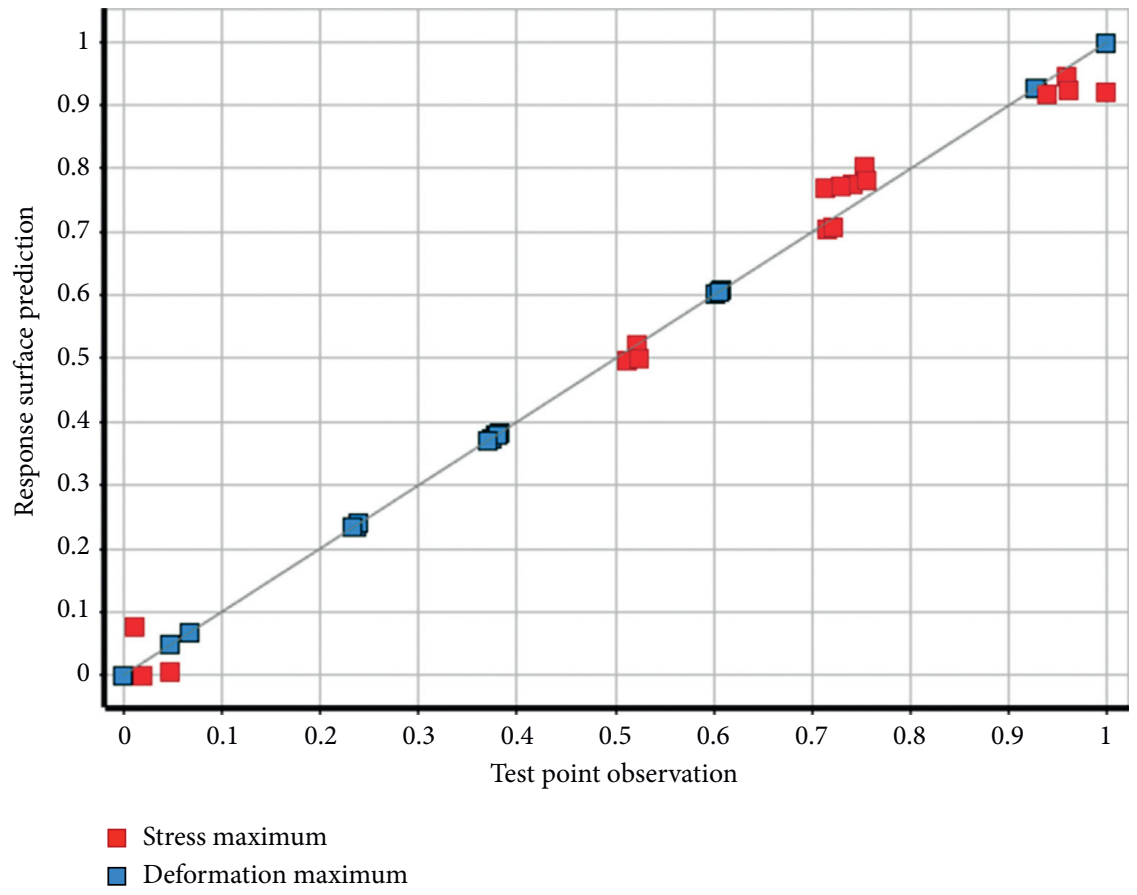

Figure 13: Predicted value and experimental value. 




(a)

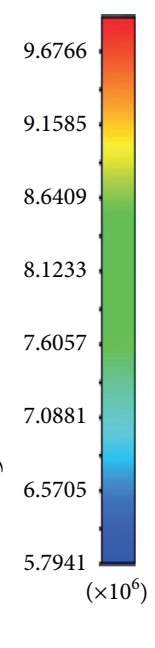

$\left.\times 10^{6}\right)$
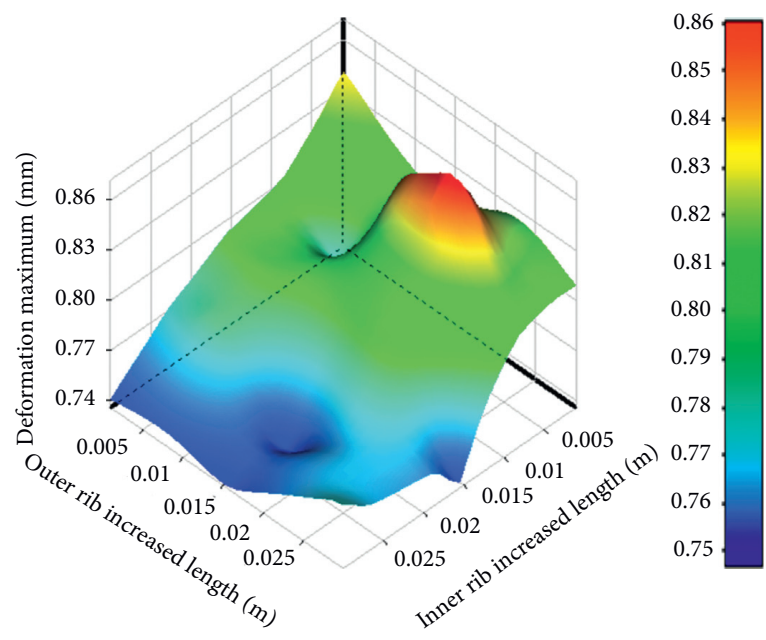

(b)

Figure 14: The response surface of the feeder's stress and deformation varying with the length of the rib-stiffened plates on both sides: (a) first principal stress response surface; (b) deformation response surface.

the outer rib-stiffened plate width by $10 \mathrm{~mm}$ should be avoided. Therefore, it was chosen as the optimal solution that the widths of the inner and outer rib-stiffened plates are both increased by $30 \mathrm{~mm}$ considering the stress and deformation comprehensively. It can be seen from Figure 14(a) that the maximum stress value after parameter optimization was $5.7941 e+6 \mathrm{~Pa}$. The maximum stress value was reduced by $40.12 \%$ according to equation (2).

\section{Conclusions}

The finite element stress analysis was carried out for four limiting conditions within one cycle of the vibrating feeder based on the inertial release method. It was found that the first principal stress of the vibrating feeder reaches the maximum when the excitation force is forward, occurring in the middle and both ends of the vibrating feeder beam.

The effect of the materials force was taken into account in the finite element analysis of the vibrating feeder. The EDEM was used to simulate the process of the vibrating feeder conveying the materials. Then, the coupling force of the material and the excitation force was obtained based on the phase characteristics of the vibrating feeder. The materials force is the largest when the vibrating feeder moves close to the highest point of vibration.

Structural optimization Schemes 1-3 were designed and compared, which, respectively, were constructing a statically indeterminate structure, increasing the width of the ribstiffened plates on the beam, and increasing the internal spacing of the beam. The maximum value reduction rates of the first principal stress of Schemes 1-3 are $-44.13 \%, 25.63 \%$, and $25.43 \%$, respectively. It was determined that increasing the width of the rib-stiffened plates was more effective than the other two schemes. Finally, the response surface method was used to optimize the width of the rib-stiffened plates, reducing the maximum value of the original first principal stress by $40.12 \%$.

\section{Data Availability}

The data used to support the findings of this study are available from the corresponding author upon request.

\section{Conflicts of Interest}

The authors declare that they have no conflicts of interest.

\section{Acknowledgments}

The authors gratefully acknowledge the support of the National Innovation Training Project (C201803813).

\section{References}

[1] Z. J. Yin, L. W. Zhang, and T. Han, "Study and development of vibratory feeder," Metallurgical Equipment (China), vol. 122, no. 5, pp. 49-54, 2010.

[2] U. Weingerl and U. Schaflinger, "Feeding of granular material on conveyer bands or chutes," Powder Technology, vol. 108, no. 1, pp. 1-5, 2000.

[3] I. Han and Y. Lee, "Chaotic dynamics of repeated impacts in vibratory bowl feeders," Journal of Sound and Vibration, vol. 249, no. 3, pp. 529-541, 2002.

[4] H. Ashrafizadeh and S. Ziaei-Rad, "A numerical 2D simulation of part motion in vibratory bowl feeders by discrete element method," Journal of Sound and Vibration, vol. 332, no. 13, pp. 3303-3314, 2013.

[5] X. X. Kong, X. J. Xi, and B. C. Wen, "Analysis of motion of the part on the linear vibratory conveyor," Journal of Northeastern University (Natural Science), vol. 36, no. 06, pp. 827-831, 2015.

[6] A. I. Afanas Ev and D. N. Suslov, "Regime parameters of autoresonance vibrating feeder with linear motor," Refractories and Industrial Ceramics, vol. 53, no. 1, pp. 9-11, 2012.

[7] L. L. Xin, J. H. Liang, and B. C. Wen, "Dynamic analysis of a vibrating conveyer with inclination in consideration of material combination coefficient," Transactions of the Chinese 
Society for Agricultural Machinery (China), vol. 40, no. 2, pp. 87-90, 2009.

[8] Z. C. Tang, Z. J. Yin, B. Chen et al., "Performance analysis of large radial equal-thickness vibratory feeder," Advanced Materials Research, vol. 139-141, pp. 2494-2497, 2010.

[9] Y. M. Zhang, C. S. Liu, X. M. He et al., "Dynamic design theory and application of large vibrating screen," Procedia Earth \& Planetary Science, vol. 144, no. 1, pp. 776-784, 2009.

[10] R. H. Su, L. Q. Zhu, and C. Y. Peng, "CAE applied to dynamic optimal design for large-scale vibrating screen," Proceedings of Acis International Symposium on Cryptography, vol. 31, no. 34, pp. 316-320, 2011.

[11] S. Baragetti, "Innovative structural solution for heavy loaded vibrating screens," Minerals Engineering, vol. 84, pp. 15-26, 2015.

[12] L.-P. Peng, C.-S. Liu, B.-C. Song et al., "Improvement for design of beam structures in large vibrating screen considering bending and random vibration," Journal of Central South University, vol. 22, no. 9, pp. 3380-3388, 2015.

[13] X. Wu, J. Wu, H. Jiang et al., "Dynamic modeling and parameters optimization of large vibrating screen with full degree of freedom," Shock and Vibration, vol. 2019, pp. 1-12, Article ID 1915708, 2019.

[14] N. Qiu and Y. K. Shyy, "Influence of inertia relief on optimal designs," in Proceedings of the Paper presented at the Aiaal issmo Multidisciplinary Analysis \& Optimization Conference, Albany, NY, USA, August 2004.

[15] L. Liao, "A study of inertia relief analysis," in Proceedings of the Paper presented at the 52nd AIAA/ASME/ASCE/AHS/ASC Structures, Structural Dynamics and Materials Conference, Denver, CO, USA, April 2011.

[16] M. F. Nelson and J. A. Wolf Jr, Use of Inertia Relief to Estimate Impact Loads, Elsevier, Amsterdam, Netherlands, 1977.

[17] K. Yan and G. D. Cheng, "Application and Improvement of inertial release method in topological optimization of structural crash-resistance," Chinese Journal of Computational Mechanics, vol. 32, no. 3, pp. 293-300, 2015.

[18] Y. Wang, Y. Luo, Z. Chen et al., "Application of inertia relief in the prediction of welding deformation for large complex structures," Journal of Ship Mechanics, vol. 20, no. 9, pp. 1147-1159, 2016.

[19] Y. Xu, R. X. Zhao, C. H. Xu et al., "Experimental study on flow arching of equal diameter ball particles in flat bottom silo," in Proceedings of the National Conference on Computational Mechanics of Granular Materials, pp. 462-467, Toledo, OH, USA, December 2012.

[20] S. S. Rao, Mechanical Vibrations, Pearson Education India, Chennai, India, 2004.

[21] Y. M. Zhao, C. S. Liu, X. M. He et al., "Dynamic design theory and application of large vibrating screen," in Proceedings of the International Conference on Mining Ence \& Technology, vol. 1, no. 1, pp. 0-784, Buffalo, NY, USA, July 2009.

[22] H. W. Liu, Mechanics of Materials -II, Higher Education Press, Beijing, China), 2011.

[23] H. Jiang, Y. S. Guan, Z. C. Qiu et al., "Dynamic and static multi-objective optimization of a vertical machining center based on response surface method," Journal of Mechanical Engineering, vol. 47, no. 11, pp. 125-133, 2011. 